\title{
Evaluación del efecto inhibidor de la enzima polifenol oxidasa en una salsa de aguacate (Persea americana) ${ }^{1}$
}

\section{Evaluation of the inhibitor effect of polifenol oxidaza enzyme in an avocado sauce (Persea americana)}

\author{
N. A. Vega, S. A. Salazar, L.T. Bautista y G. E. Muñoz
}

Recibido: agosto 21 de 2019 - Aceptado: junio 20 de 2020

\begin{abstract}
Resumen-Esta investigación busca estimar la inhibición de la enzima polifenol oxidasa en una pasta de Persea Americana variedad Hass por medio de la cáscara de cebolla (Allium cepa) de dos especies bulbo blanco y rojo sometidas a dos tratamientos térmicos de $100^{\circ} \mathrm{C}$ y $115{ }^{\circ} \mathrm{C}$, con el fin de inhibir la polifenol oxidasa la cual convierte los fenoles en quinonas, causando el pardeamiento enzimático; para ello se realizaron diferentes tratamientos, utilizando la aplicación de un agente inhibidor a concentraciones $y$ tiempos diferentes buscando aplicaciones ideales a tiempos determinados, se midieron las absorbancias de cuatro tratamientos, 1:0, 1.1, 1.2 y 2.1 respectivamente correspondientes a la enzima $y$ al agente inhibidor, como resultados de la evaluación la actividad enzimática, se logró inhibición completa para una concentración 1:2 para la cebolla de bulbo blanco a temperatura de $100{ }^{\circ} \mathrm{C}$ en relación enzima sustrato, con la cebolla roja la inhibición fue variable para cada tratamiento.
\end{abstract}

Palabras claves--Aguacate, Pardeamiento enzimático-Polifenol Oxidasa Peroxidasa, Quercetina

${ }^{1}$ Proyecto de investigación apoyado por la Universidad Francisco de Paula Santander.

N. Vega, Universidad Francisco de paula Santander, Cúcuta Colombia email: nelsonalfosnovc@ufs.edu.co.

S. Salazar, Universidad Francisco de paula Santander, Cúcuta email: salzar663@hotmail.com.

T. Bautista, Universidad Francisco de paula Santander, Cúcuta Colombia email: Tatadrums@hotmail.com.

G. Muñoz, Universidad Francisco de paula Santander, Cúcuta Colombia email: gloriaflorez1511@gmail.com

Como citar este artículo: Vega, N, Salazar, S., Bautista, T., Muñoz G. Evaluación del efecto inhibidor de la enzima polifenol oxidasa en una salsa de aguacate (persea americana.), Entre Ciencia e Ingeniería, vol. 14, no. 27, pp. 58-62, enero-junio 2020. DOI: https://doi.org/10.31908/19098367.1775.

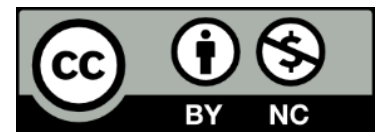

Attribution-NonCommercial 4.0 Intenational (CC By-NC 4.0
Abstract-This research is to rate the inhibition of Polyphenol Oxidase enzyme in a paste made of avocado (Persea Americana Mill) variety Hass through an onion peel (Allium Cepa) from two sorts of bulbs, white one and red one; exposed to two thermic treatments over $100^{\circ} \mathrm{C}$ and $115^{\circ} \mathrm{C}$ aiming to inhibit the effect of Polyphenol Oxidase which convert Phenols into Quinones causing the enzymatic browning. So that it implied to perform diversity of treatments, employing an inhibitor agent on different concentrations and periods of time in order to determine the ideal applications on determined times which required measuring the absorbance in four treatments, 1:0, 1.1, 1.2 and 2.1 respectively corresponding to the enzyme and the inhibitor agent, as a result of the enzymatic activity test a complete inhibition was reached for a concentration of $1: 2$ for the white bulb onion case in temperature of $100^{\circ} \mathrm{C}$ related to enzyme substratum; in red bulb onion case the inhibition varied for each treatment.

Keywords-Polyphenol Oxidase, Enzymatic, Browning, Quercetin Avocado Peroxidase.

\section{INTRODUCCIÓN}

$\mathbf{Z}^{\mathrm{L}}$ aguacate (Persea americana) es una fruta - correspondiente a la familia de las lauráceas, cuyo origen tuvo lugar en el centro y este de México, y partes altas de Guatemala [1], [2]. La evidencia más antigua del consumo de aguacate, fueron encontrados en una cueva en Coxcotlan región de Tehuacán, puebla [3]. En Colombia se han estudiado las variedades nativas desde hace más de 40 años permitiendo evaluar su comportamiento y producción [4], encontrándose 10 especies de gran importancia económica. El aguacate es una fruta de gran contenido nutricional [5] [6], utilizada hoy en día como un alimento saludable en todo el mundo. Además, tiene actividad antimicrobiana contra Mycobacterium tuberculosis [7], se cultiva principalmente para el consumo local, con gran potencial exportador, como frutas frescas y procesadas. La problemática en la industrialización del aguacate es el deterioro que se manifiesta en un rápido obscurecimiento. Por sus características fisicoquímicas, es una materia prima difícil de procesar y almacenar [8], esto se debe a la presencia de diversas enzimas 
que influyen en el proceso de conservación y vida útil del producto, una de ellas es la enzima polifenol oxidasa (PFO) causante del pardeamiento enzimático, la polifenol oxidasa (PFO) es una metaloenzima que se encuentra ampliamente distribuido en plantas y hongos, en su estructura contiene dos átomos de cobre en el sitio activo que catalizan dos tipos de reacciones usando $\mathrm{O}_{2}$ como agente oxidante: (a) la ohidroxilación de monofenoles para producir o-difenoles (actividad monofenol monoxigenasa E.C. 1.14.18.1); y (b) la posterior oxidación de o-difenoles a o-quinonas.[9], [10], Confinadas generalmente en los plastidios de células vegetales siendo liberadas posteriormente en el citoplasma ocasionando daño a nivel celular y tisular [11], Lo que con lleva a cambios en las propiedades organolépticas y pérdidas en los valores nutricionales [12], haciéndola susceptible a diversas variables como el oxígeno, el pH y la temperatura, que intervienen en su velocidad de reacción y a su vez, se ve influenciada por el efecto de otras enzimas presentes en la pulpa, lo que dificulta su conservación, y así mismo ocasiona una afectación en su vida útil. Algunos trabajos de PPO planteados por [13], [14], [15], [16], han descrito la presencia de actividad PPO en fracciones particuladas y solubles del mismo tejido [17]. Es por ello que en la industria de alimentos, la evidencian de las diferencias en las propiedades cinéticas que afectan la actividad de la PFO que puede ser evitada usando tratamientos térmicos [18], lo que conllevaría afectar algunas de sus características. La PFO a medida que aumenta la temperatura incrementa su actividad enzimática [19], limitando la aplicación de diversos métodos de conservación y creando la necesidad de investigar métodos que permitan la inhibición de la enzima sin afectar sus propiedades. Esta investigación pretende estimar la inhibición de la enzima polifenol oxidasa en una pasta de aguacate (Persea Americana) variedad Hass por medio de la cáscara de cebolla (Allium cepa) de dos especies bulbo blanco y de bulbo rojo, que gracias a su composición rica en flavonoides como la quercetina, actúa con un alto poder antioxidante natural [20], [21], [22], [23] que interrumpe la interacción que existe en el sustrato enzimático, evitando la reacción oxidativa de la enzima [24], Para ello será sometida a tratamientos térmicos de 100 y 115 ${ }^{\circ} \mathrm{C}$ para evaluar la actividad enzimática, teniendo en cuenta la concentración ideal y el control durante su almacenamiento en factores como el color, $\mathrm{pH}$ y temperatura, con la finalidad de emprender en términos de investigación nuevas alternativas en métodos de conservación natural.

\section{MATERIALES Y MÉTODOS}

Se realizó la evaluación de un agente inhibidor a base de cáscara de cebolla (Allium cepa) de bulbo rojo $(3.64 \mathrm{Kg}$ ) y de bulbo blanco $(2.75 \mathrm{Kg})$ a temperaturas de $115^{\circ} \mathrm{C}$ y $100^{\circ} \mathrm{C}$ en una salsa de aguacate (Persea americana) de la variedad Hass como materia prima. Para ello se utilizaron seis tratamientos del agente inhibidor como se expresan en la Tabla 1.

\section{A. Elaboración del agente inhibidor}

El agente inhibidor se elaboró a partir de los residuos de cascaras de cebolla seleccionando las de menor contenido de humedad, sometiéndose a un proceso de lavado en solución de hipoclorito de sodio al $99 \%$ y posterior secado de las mismas, para ser llevadas a un proceso de molienda y obtener una pasta fina, la cual se sometió finalmente a calentamiento a una temperatura de $100^{\circ} \mathrm{C}$ a un tiempo determinado.

TABLA I

\begin{tabular}{|c|c|c|c|c|c|c|}
\hline Materiales & T0 & $\mathrm{T} 1$ & $\mathrm{~T} 2$ & T3 & $\mathrm{T} 4$ & T5 \\
\hline Aguacate & $100 \%$ & $99 \%$ & $99 \%$ & $99 \%$ & $97 \%$ & $99.95 \%$ \\
\hline $\begin{array}{l}\text { Cáscara de } \\
\text { cebolla } \\
\text { blanca }\end{array}$ & 0 & $1 \%$ & $1 \%$ & 0 & 0 & 0 \\
\hline $\begin{array}{l}\text { Cáscara de } \\
\text { cebolla roja }\end{array}$ & 0 & 0 & 0 & $1 \%$ & $3 \%$ & 0 \\
\hline $\begin{array}{l}\text { Ácido } \\
\text { Ascórbico }\end{array}$ & 0 & 0 & 0 & 0 & 0 & $0.02 \%$ \\
\hline $\begin{array}{l}\text { Ácido } \\
\text { Cítrico }\end{array}$ & & & & & & $0.01 \%$ \\
\hline Benzoato & & & & & & $0.02 \%$ \\
\hline Total & $100 \%$ & $100 \%$ & $100 \%$ & $100 \%$ & $100 \%$ & $100 \%$ \\
\hline
\end{tabular}

\section{B. Interacción agente inhibidor polifenol oxidasa}

La extracción de la enzima Polifenol oxidasa presente en la salsa de aguacate, se trabajó siguiendo la metodología descrito por [25] con modificaciones [26], Para ello se utilizó la solución Tris $\mathrm{HCl} 100 \mathrm{mM}$ (pH 7.1), con $1 \%$ de polivinil pirrolidona (PVP). Para la evaluación de la actividad enzimática se empleó el Catecol $20 \mathrm{mM}$ como sustrato, disuelto en una solución de Tris $\mathrm{HCl}$, midiendo el cambio de absorbancia a $400 \mathrm{nM}$ en $0,2 \mathrm{ml}$ de sobrenadante del extracto enzimático al adicionarse $3 \mathrm{ml}$ de Catecol a temperaturas entre $22^{\circ}$ y $29^{\circ} \mathrm{C}$.

\section{Evaluación de la actividad enzimática}

Se evaluó la actividad enzimática de la Polifenol en respuesta de la adición del sobrenadante del agente inhibidor a concentraciones de $(1: 1)$, (1:2) y (2:1) en relación enzima: sustrato. Los tiempos de la lectura que se tuvieron en cuenta fueron de $0,1,3,5,7$ y 10 minutos respectivamente tiempo en el cual se analizó la reacción de la enzima PFO en cada tratamiento.

\section{RESULTADOS Y DISCUSIONES}

El agente inhibidor se realizó con residuos de cascara de cebolla (Allium cepa) de bulbo rojo y de bulbo blanco, para ello se realizaron pruebas de medición de absorbancia a muestras con diferentes concentraciones, realizadas por triplicado, lo que permitieron medir la existencia o no del efecto inhibidor. Las concentraciones tomadas para su análisis fueron de $1: 0,1.1,1.2$ y 2.1 respectivamente correspondiente a la enzima y a agente inhibidor, tomándose lectura en seis momentos a ( $\mathrm{t} 0$ : tiempo $0, \mathrm{t} 1$ : al minuto, $\mathrm{t} 3$ : tres minutos, $\mathrm{t} 5$ : cinco minutos, $\mathrm{t} 7$ : siete minutos y t10: diez minutos), calculándose el promedio de absorbancias de las tres replicas. Para el primer tratamiento se consideró la muestra sin agente inhibidor (concentración 1:0), la lectura inicial o momento cero como patrón o blanco de referente para observar el cambio de absorbancia. El dato registrado con la concentración 1:0 y con cada uno de los cuatro tratamientos estuvo entre 2.875 a 2.876 , indicando una variación mínima en los valores, de igual forma, los datos obtenidos al minuto $\mathrm{t} 1, \mathrm{t} 3, \mathrm{t} 5, \mathrm{t} 7$ y t10, se percibieron constantes, reiterándose la uniformidad de estos y variación mínima entre la lectura inicial (t0) y la final (t10) de 0.846 a 0.848 (Fig. 1). 


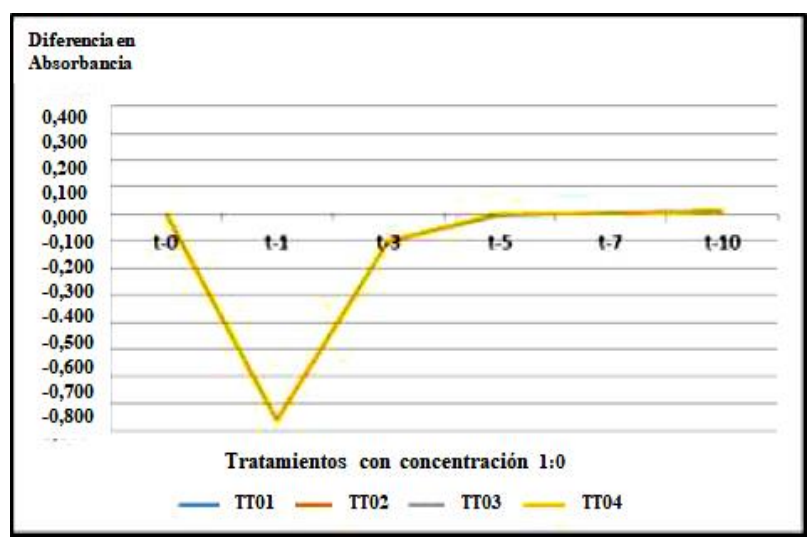

Fig. 1. Lectura en seis momentos (minutos) de la absorbancia registrada para los cuatro tratamientos Evaluados en ausencia de agente inhibidor. *

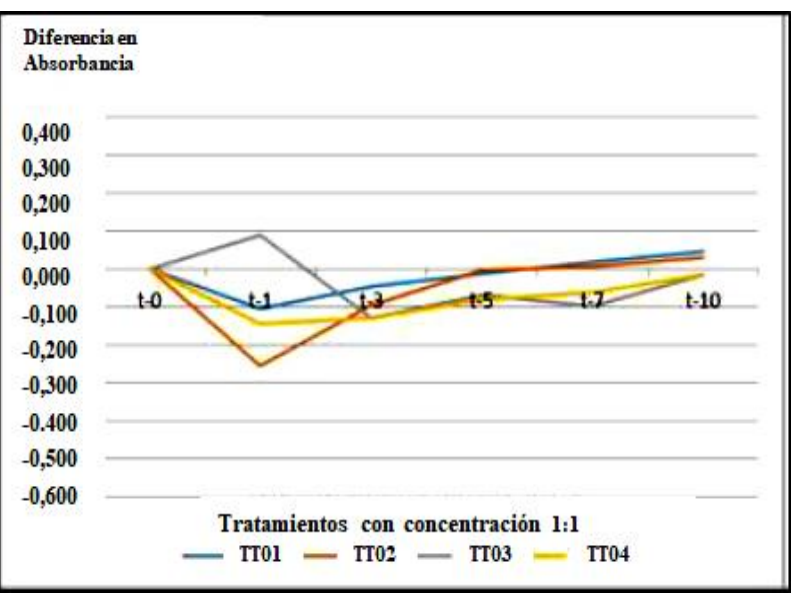

Fig. 2. Lectura de la absorbancia para los cuatro tratamientos evaluados a una concentración con el agente inhibidor de 1:1.

La lectura negativa indica que el dato al minuto diez (t10) fue menor al dato registrado al momento cero (t0). Al evaluar las muestras a una concentración 1:1 se evidencio mayor uniformidad en las lecturas graficadas a partir del uso de cáscara de cebolla bulbo blanco a $115^{\circ} \mathrm{C}(\mathrm{T} 1)$ y cáscara de cebolla bulbo rojo a $100^{\circ} \mathrm{C}$ (T4) (Fig. 2).

TO: tratamiento $(\mathrm{T})$. Las muestras evaluadas a una concentración de (1:2), la mayor uniformidad en los datos se registró a partir del uso de cáscara de cebolla bulbo blanco a $115^{\circ} \mathrm{C}(\mathrm{T} 1)$, seguido de cáscara de cebolla bulbo rojo a $115^{\circ} \mathrm{C}$ (T3) y $100^{\circ} \mathrm{C}$ (T4). (Fig. 3).

El tratamiento 1 de concentración 1:2, evidenció durante los seis momentos una lectura de $3.00 \pm 0.00$ de absorbancia, indicando una inhibición total o cercana al $100 \%$ de la PFO presente en la salsa de aguacate (Persea americana) variedad Hass. En relación con las muestras evaluadas a una concentración de (2:1), la mayor uniformidad en los datos se registró a partir del uso de cáscara de cebolla bulbo blanco a $115^{\circ} \mathrm{C}$ (T1) y cáscara de cebolla bulbo rojo a $115^{\circ} \mathrm{C}$ (T3). (Fig. 4).

De acuerdo con las lecturas de tiempos t10 menos el registro t0, la cáscara de cebolla de bulbo blanco a $115^{\circ} \mathrm{C}(\mathrm{T} 1)$ indicó un mayor efecto de inhibición en cualquiera de las tres concentraciones; seguido se puede plantear el uso de cáscara de cebolla bulbo rojo a $100^{\circ} \mathrm{C}$ (T4) en una concentración de 1:2 o cáscara de cebolla bulbo rojo a $115^{\circ} \mathrm{C}$ (T3) en una concentración de $1: 1$ y 2:1.

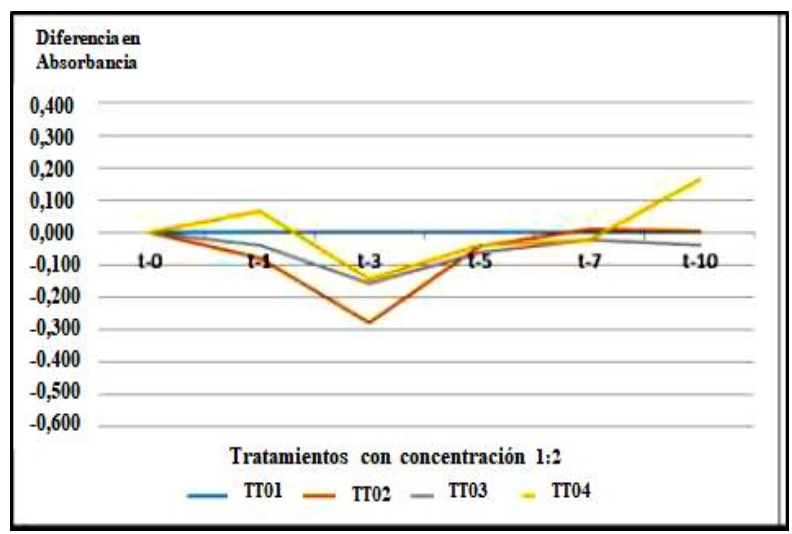

Fig. 3. Lectura de la absorbancia para los cuatro tratamientos evaluados a una concentración con el agente inhibidor de 1:2.

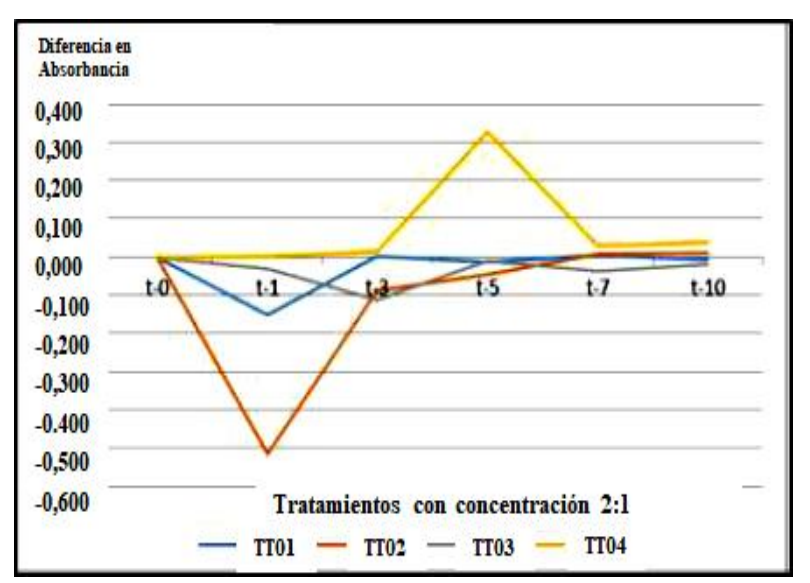

Fig. 4. Lectura de la absorbancia para los cuatro tratamientos evaluados a una concentración con el agente inhibidor de 2:1.

La PPO mostró mayor sensibilidad a los cambios de temperatura, resultado similar reportaron [27] en su estudio de la estabilidad térmica de la PPO en la batata colorada. Por otra parte, estudios realizados por [28], encontraron que la PPO presente en manzana disminuye un $40 \%$ con un tratamiento a $50^{\circ} \mathrm{C}$ durante $20 \mathrm{~min}$ y en la pera a $70^{\circ} \mathrm{C}$ por $30 \mathrm{~min}$. Lo que difiere con la presente investigación. A temperaturas superiores a $50{ }^{\circ} \mathrm{C}$ se muestra la poca estabilidad de la enzima (Polifenol Oxidasa), al igual que por largos periodos de almacenamiento y la manipulación en condiciones en las cuales la enzima pierde su actividad [29] [30]. La variación en la lectura de absorbancia a partir del uso de cáscara de cebolla bulbo blanca a $100^{\circ} \mathrm{C}$ (T2), fue mayor en todos los casos, estimándose el menor efecto inhibitorio (Tabla II).

La evaluación de los tratamientos realizados a diferentes concentraciones identificó que el tratamiento T1 obtuvo mayor efecto de inhibición en todas las concentraciones y una inhibición total a concentración 1:2, por lo que se evidencia que el tratamiento de cebolla blanca sometida a temperatura de $115^{\circ} \mathrm{C}$ es el tratamiento con mayor efecto de inhibición. La variación de la temperatura es un factor que afecta según la especie ya que, la naturaleza proteica de las enzimas su estructura terciaria se ve comprometida cuando se alcanzan elevadas temperaturas, lo que conduce a la pérdida de actividad catalítica [31], para la cebolla blanca su mayor efecto inhibitorio se da al someterse a una temperatura alta. Sin embargo, en la especie de cebolla roja se observa un efecto contrario, a pesar de ello la cebolla roja a $100^{\circ} \mathrm{C}$ a 
concentración 1:2 obtuvo una mejor inhibición con respecto a la cebolla blanca a la misma temperatura la cual la hace competitivo para estos tratamientos es decir que los tratamientos según el comportamiento enzimático que obtendrán mejor inhibición serán los compuestos por cebolla blanca a $115^{\circ} \mathrm{C}$ y cebolla roja a $100^{\circ} \mathrm{C}$.

TABLA II.

Relación De Las Concentraciones Vs la Absorbancia Por Cada TRATAMIENTO. [2]

\begin{tabular}{|c|c|c|c|c|}
\hline & \multicolumn{4}{|c|}{ Concentración } \\
\cline { 2 - 5 } & {[] $1: 0$} & {[] $1: 1$} & {[] $1: 2$} & {[] $2: 1$} \\
\hline T1 & $-0,848$ & -0.098 & 0.000 & -0.161 \\
\hline T2 & $-0,846$ & $-0,317$ & $-0,389$ & $-0,629$ \\
\hline T3 & $-0,846$ & $-0,224$ & $-0,318$ & $-0,206$ \\
\hline T4 & $-0,847$ & $-0,431$ & 0,021 & 0,407 \\
\hline
\end{tabular}

\section{CONCLUSIONES}

Se analizó la acción del agente inhibidor sobre la enzima Polifenol oxidasa extraída de aguacate (Persea americana) variedad Hass con lo cual se pudo inferir que la cáscara de la cebolla (Allium cepa) de bulbo rojo y de bulbo blanco posee capacidad inhibidora sobre la enzima Polifenol oxidasa, donde la especie de cebolla blanca a temperatura $115^{\circ} \mathrm{C}$ obtuvo mayor inhibición con respecto a los demás tratamientos. De acuerdo con las lecturas de las absorbancias se logró determinar que la inhibición total de la enzima se logró a una concentración 1:2, con el tratamiento de cebolla roja sometida a temperatura de $100^{\circ} \mathrm{C}$, se obtuvo una inhibición casi total de la enzima. Sin embargo, por las características de lectura no fueron constantes para obtener porcentajes exactos de inhibición de cada tratamiento, afirmándose que la cebolla es un agente inhibidor y se evidencia que el aumento de la temperatura aplicado a la cáscara de cebolla influye de forma positiva para la inhibición de la enzima polifenol oxidasa.

\section{AGRADECIMIENTOS}

A la Universidad Francisco de Paula Santander por su valiosa colaboración.

\section{REFERENCIAS}

[1] Williams, LO (1977). Los aguacates, una sinopsis del género Persea, subg. Persea Botánica Económica , 31 (3), 315-320.

[2] Knight, R.J. (2002). History, distribution and uses. In: The Avocado: Botany, Production and Uses (edited by A.W. Whiley, B. Schaffer, \& B.N. Wolstenholme). Pp 1-314, Wallingford, UK: CAB International

[3] Smith, C. E. (1966). Archeological evidence for selection in avocado. Economic Botany, 20(2), 169-175.

[4] Ríos-Castaño, D. (2003). Variedades De Aguacate Para El Trópico: Actas V Congreso Mundial Del Aguacate, 143-147

[5] Serra Bonvehi, J., Ventura Coll, F., Orantes Bermejo, J. (2019). Characterization of avocado honey (Persea americana Mill.) produced in Southern Spain. Food Chemistry. 287, 214-221.

[6] Wang J Wang A Zang X Tan L Xu B et. al. 2019. Physicochemical, functional and emulsion properties of edible protein from avocado (Persea americana Mill.) oil processing by-products. Food Chemistry. 288, 146-153.

[7] Lu Y Chang H Peng C Lin C Chen I. Secondary metabolites from the unripe pulp of Persea americana and their antimycobacterial activities. Food Chemistry. 135, 2904-2909
[8] Corrales-García J. (1991). Experiencias y problemática de la industrialización del aguacate. Memorias del Seminario Internacional del Aguacate. Post cosecha y Comercialización. México. p.p. 64-71

[9] Guillou, N. 2012. Mecanismos Y Efectos Asociados A Procesos De Oxidación De Compuestos Fenólicos En Vinos. Trabajo de grado. Facultad de Ciencias Agronómicas. Universidad de Chile, Santiago.

[10] Hatzakis, E., Mazzola, E., Shegog, R., Ziegler, G., Lambert, J. (2019). Perseorangin: A natural pigment from avocado (Persea americana) seed. Food Chemistry. 293, 15-22.

[11] Mayer, A. (2006). Polyphenol oxidases in plants and fungi: Going places? A review. Phytochemistry, 67(21), 2318- 2331. doi: 10.1016/j.phytochem.2006.08.006

[12] Queiroz C, Ribeiro da Silva A, Mendes-Lopes M, Fialho E, Valente Mesquita V. 2011. Polyphenol oxidase activity, phenolic acid composition and browning in cashew apple (Anacardium occidentale, L.) after processing. Food Chemistry, 125 (1): 128-132

[13] Casado-Vela, J., Sellés, S., and Bru, R. 2005. Proteomic approach to blossom-end rot in tomato fruits (Lycopersicon esculenrum M.). Proteomics 5(10): 2488-2496.

[14] Ding, CK, Chachin, K., Ueda, Y. y Wang, CY (2002). Inhibición del pardeamiento enzimático de níspero por compuestos de sulfhidrilo. Química de los alimentos, 76 (2), 213-218.

[15] Gandía-Herrero, F., Jiménez-Atiénzar, M., Cabanes, J., GarcíaCarmona, F. and Escribano, J. 2005a. Differential activation of a latent polyphenol oxidase mediated by sodium dodecyl sulfate. Journal of Agricultural and Food Chemistry 53(17): 6825-6830.

[16] Sellés-Marchart, S., Casado-Vela, J., and Bru-Martínez, R. 2006. Isolation of a latent polyphenol oxidase from loquat fruit (Eriobotrya japonica Lindl.): kinetic characterization and comparison with the active form. Archives of Biochemistry and Biophysics 446(2): 175-85.

[17] Morante Carriel, J., Agnieszka-Obrebska, A., Bru, R., Carranza Patiño, M., Pico-Saltos, R., \& Nieto Rodriguez, E. (2014). Distribución, localización e inhibidores del polifenol oxidasas en frutos y vegetales usados como alimento.

[18] Marshall, MR, Kim, J. y Wei, C. (2000). Dorado enzimático en frutas, verduras y mariscos. FAO, Roma, 49.

[19] Hernández, E., \& Briceño, L. (2009, December). Evaluación del pardeamiento enzimático durante el almacenamiento en congelación del puré de palta (Persea americana Mill) Var. Hass. In Anales Científicos (Vol. 70, No. 4, pp. 24-35).

[20] Gertz., Christian.; Klostermann., Sabine.; Kochhar., Parkash. 2000. Testing and comparing oxidative stability of vegetable oils and fats at frying temperature. European Journal of Lipid Science and Technology, Vo. 102, 543-551

[21] Ravichandran R Rajendran M Devapiriam D. (2014). Antioxidant study of quercetin and their metal complex and determination of stability constant by spectrophotometry method. Food Chemistry. 146, 472-478.

[22] Cruz-Zúñiga J Soto-Valdez H Peralta E Mendoza-Wilson A RoblesBurgueño M et. al. 2016. Development of an antioxidant biomaterial by promoting the deglycosylation of rutin to isoquercetin and quercetin. Food Chemistry. 204, 420-426.

[23] Nile S Nile A Keum Y Sharma K. (2017). Utilization of quercetin and quercetin glycosides from onion (Allium cepa L.) solid waste as an antioxidant, urease and xanthine oxidase inhibitors. Food Chemistry. 235, 119-126.

[24] Martínez-Flórez, S., González-Gallego, J., Culebras, J. M., \& Tuñón, M (2002). Los flavonoides: propiedades y acciones antioxidantes. Nutr Hosp, 17(6), 271-278.

[25] Lamikanra, O. 1995. Enzymatic browning of Muscadine grapes products. pp. 166-177. In: Enzymatic Browning and its Pre vention, LEE, C.L.; WHITAKER, J. R. (eds). ACS. Washington, USA.

[26] Balois-Morales, R., Colinas-León, M. T., Peña-Valdivia, C. B., ChávezFranco, S. H., \& Alia- Tejacal, I. (2007). Sistema De Estrés Oxidativo, Fenoles-Polifenol Oxidasa-Peroxidasa, De Frutos De Pitahaya (, 13(2), 115-120.\}

[27] Gao, L., Yuan, Y., Li, YC y Wei, YX (2014). Estudio sobre la actividad del polifenol oxidasa en diferentes partes de la batata morada. En Advanced Materials Research (Vol. 898, pp. 149-

[28] Gasull, E., \& Becerra, D. (2006). Caracterización de Polifenoloxidasa Extraída de Pera (cv. Packam's Triumph) y Manzana (cv. Red Delicious). Información tecnológica, 17(6), 69-74.

[29] Constabel, C. P., Yip, L., Patton, J. J., \& Christopher, M. E. (2000). Polyphenol oxidase from hybrid poplar. Cloning and expression in response to wounding and herbivory. Plant physiology, 124(1), 285-296 
[30] Mazzafera, P., \& Robinson, S. P. (2000). Characterization of polyphenol oxidase in coffee. Phytochemistry, 55(4), 285-296

[31] Pérez-Gálvez, R., Almécija, M. C., Espejo, F. J., Guadix, E. M., \& Guadix, A. (2011). Bi-objective optimisation of the enzymatic hydrolysis of porcine blood protein. Biochemical Engineering Journal, 53(3), 305-310.

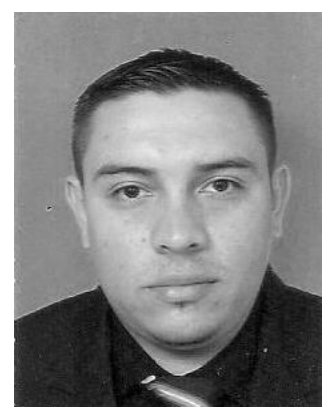

Nelson Alfonso Vega Contreras. Nacido en Cúcuta Colombia el 2 de febrero de 1983 Magister en Practica Pedagógica, licenciado en biología y química y especialista en bioquímica es docente del Departamento de Ciencias del medio ambiente de la Universidad Francisco de Paula Santander. Desarrolla sus trabajos de investigación en cuanto al uso del portafolio como herramienta didáctica para el desarrollo de competencias en los estudiantes, así como también en procesos bioquímicos ha publicado artículos científicos, nacionales e internaciones sobre las prácticas pedagógicas y procesos bioquímicos. ORCID: https://orcid.org/0000-00025013-2146.

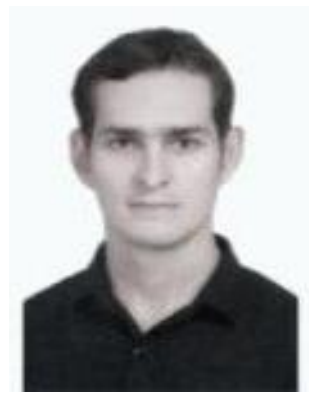

Seir Antonio Salazar Mercado, Magister en Practica Pedagógica, biólogo de la universidad de Pamplona es docente del Departamento de Biología de la Universidad Francisco de Paula Santander. Desarrolla sus trabajos de investigación en cuanto a la implementación de herramientas didácticas en la educación superior y en procesos biológicos. $\mathrm{Ha}$ participado en eventos y ha publicado artículos científicos, nacionales e internaciones sobre las prácticas pedagógicas y procesos biológicos. ORCID: https://orcid.org/00000002-3287-703X.

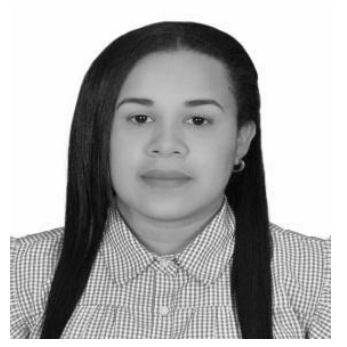

Linda Tatiana Bautista, nació en Villa Del Rosario, Norte De Santander, Colombia, el 15 de abril del 1995. Inicio sus estudios de Ingeniería Agroindustrial en el 2012 en la universidad Francisco de Paula Santander, participo en los semilleros de investigación "SINARBA" y "SINPROBIO"; en el 2016 fue ponente en la Universidad Cooperativa de Colombia, sede Arauca, en el II Encuentro Binacional de Jóvenes Investigadores; En el 2018 se gradúa con mérito académico "Medalla de plata". Ejerció laboralmente en una Planta tostadora en Rescate "OMA". Actualmente es líder de calidad en Makro Supermayorista, estudia para ser auditor interno y manejar el idioma inglés. Sus principales temas de interés son la calidad, ciencia, tecnología e innovación alimentaria.

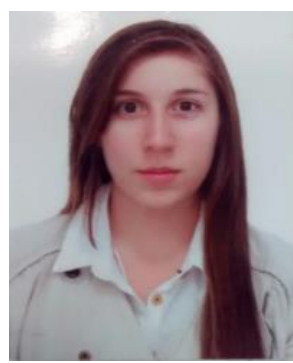

calidad alimentaria.
Gloria Muñoz. Nacida en Cúcuta, Norte de Santander, Colombia, el 15 de noviembre de 1993. Se graduó de bachiller en el colegio Luis Carlos Galán Sarmiento, inicio sus estudios como ingeniera agroindustrial en el 2012 en la Universidad Francisco de Paula Santander, participo en los semilleros de investigación SINARBA y SIMEDPA en el 2018 obtiene su título profesional. Ejerció en una empresa colombiana perteneciente a la industria de cereales y actualmente ejerce en una industria láctea. Entre sus campos de interés está la 